\title{
Computer-based methodology laboratories: 1. An undergraduate course in experimental psychology
}

\author{
MICHAEL PERONE \\ West Virginia University, Morgantown, West Virginia
}

\begin{abstract}
At West Virginia University, microcomputers are used in the laboratory sections of a conventional undergraduate course on research methods in psychology. IBM-compatible computers and QuickBasic programs are used to duplicate the functions of equipment that is less reliable and, taken collectively, more expensive-equipment such as stimulus projectors, pursuit rotors, timers, and mirror tracing stands. The computer systems increase the speed and efficiency of data collection, reduce errors of procedure and measurement, and implement numerous steps to standardize procedures and limit extraneous influences. As a consequence, uncontrolled variability is minimized, and the opportunity for meaningful research outcomes is maximized. The laboratory can be expanded with software alone, without the expense of additional hardware.
\end{abstract}

Conventional courses in psychological research methods, which consider such basic issues as observation and measurement, experimental design, and analysis and interpretation of data, pose a challenge for students and instructors alike. When the course is taught early in the undergraduate curriculum, as is often the case, the students may know little about substantive areas of psychology and may lack desirable quantitative skills, particularly if a statistics course is not a prerequisite. The effect of these deficiencies is to fix the starting point at an elementary level. Nevertheless, the goal can still be high, because the students are expected to go on to demanding upperlevel courses that require a sophisticated understanding of research methods.

This scenario is not unusual. At West Virginia University, the sole prerequisite for the course in methodology is introductory psychology, and students enroll as early as their freshman year. Although the students have limited backgrounds, the instructors in this course are able to cover much ground, owing largely to the use of microcomputers in the laboratory sections. My purpose in this paper is to describe the objectives of the laboratory part of the course and the role that the computers play in it.

\section{LABORATORY OBJECTIVES}

The course is a 3-credit introduction to research methods, with an emphasis on experimental methods. As

\footnotetext{
The software described here was prepared in collaboration with Barbara J. Kaminski and supported by a Summer Salary Grant from the WVU College of Arts and Sciences. Barbara Metzger performed above and beyond the call of duty in preparing the figures. Correspondence should be sent to Michael Perone, Department of Psychology, West Virginia University, P. O. Box 6040, Morgantown, WV 26506-6040.
}

many as 160 students are enrolled each semester. They attend two lectures per week in a large hall, but the class is divided into small groups of 16 for two laboratory sessions per week. The laboratory sessions are conducted by graduate teaching assistants. All of the class meetings last $50 \mathrm{~min}$.

The laboratory sessions are designed to give the students first-hand experience with a range of topic areas, research designs, and methods of data analysis. In recent semesters the projects have involved issues in psychometric theory, psychophysics, operant conditioning, and perceptual adaptation. The projects are selected to illustrate research designs in four major categories: correlational studies, within-group experiments, small- $N$ experiments, and factorial experiments. In the analysis of the data, considerable emphasis is placed on visual inspection of graphs, where relations among variables can be depicted concretely and grasped readily. Statistical descriptions come next, in the form of measures of central tendency and dispersion, correlations, and simple linear regression. In the group experiments, inferential tests are introduced in the form of $t$ tests for paired measures and factorial analysis of variance. No attempt is made to achieve the breadth or depth of a statistics course; rather, the objective is to provide a firm grounding in the logic of statistical inference and experience in using and interpreting some popular tests.

Each project follows a common progression. After background reading is assigned and discussed in class, behavior is observed and measured, and the resulting data are used to generate graphs and descriptive statistics. Patterns evident here are studied further with the aid of inferential tests. Integration of these various sources of information gives rise, at last, to interpretations and conclusions bearing on the issues or hypotheses that motivated the research. 
It is critical that students have direct, personal experience with the early steps in which behavioral observations are translated into numerical and graphical terms. Exercises in which students are given data without observing the underlying behavior do not fit this approach, and therefore no simulations are used (for a discussion of the limitations of simulated data in methodology courses, see Eamon, 1986). Instead, all of the projects involve the observation of real behavior, with the students themselves serving alternately as investigator and subject. Computers assist in the process by taking the place of traditional, but less reliable and more expensive, equipment such as pursuit rotors, mirror tracing stands, precision timers, and stimulus projectors. Once the data have been collected, the students prepare the graphs by hand. This level of participation makes it easy to see how behavior can be represented in quantitative terms. Although such skill may be taken for granted in advanced students, it is not prevalent among beginners. The effort to shape the skill is worthwhile, because it is essential if students are to grasp increasingly abstract representations of behavior in the form of correlation coefficients, $F$ ratios, probability values, and so forth.

\section{HARDWARE}

The computers were selected in the summer of 1986 , with the goal of obtaining a reasonably powerful, fast, and up-to-date system as cheaply as possible. The Apple Macintosh line had to be eliminated on the basis of cost, and the Apple II was eliminated because its obsolescence seemed imminent. Of the remaining competitors, only IBM and IBM-compatibles were seriously considered. Final approval went to the Tandy $1000 \mathrm{SX}$, an XT-compatible which, at the time, was the latest development in the Tandy 1000 series. Each system operates at $7.16 \mathrm{mHz}$ and has $384 \mathrm{~K}$ of RAM, two 5.25 -in. disk drives, a color graphics video adaptor (CGA), a monochrome monitor, and a joystick. The two drives allow student data to be stored separately from system and program files. The joystick extends the range of behavior that can be studied beyond that accessible through the use of a keyboard alone. The laboratory is equipped with eight systems, one per pair of students. An additional system is reserved for the instructor's use in course administration, test construction, and program development.

\section{SOFTWARE}

Because computer-assisted research has become routine in experimental psychology, the ability to program computers for use in real-time laboratory applications is common among faculty and graduate students. In this context, it was only natural to develop the software within our department, in collaboration with a graduate teaching assistant. Although commercial software was consid- ered, most of the programs were not suitable because they merely simulated data. A few programs that were suitable, such as Bainbridge's (1986) unit on memory scanning, were rejected because they had to be bought in packages dominated by simulations.

The first versions were prepared and tested during the spring 1987 semester; programs were added until the package was essentially complete, in time for the fall 1987 semester. Of course, refinements and extensions have been introduced since then. Besides programs to collect and analyze data, the package includes a system of menus and two simple management routines (one calibrates the joystick; the other ensures that data files are saved to subdirectories associated with the individual laboratory sections). The programs are compiled in QuickBasic 3.0, which provides executable modules that are both speedy and resistant to tampering by the students.

Development of the software was guided by several considerations. One major objective was to reduce the tedium that often accompanies laboratory procedures. With traditional equipment, much of the work must be done manually, and students may have to juggle stimulus materials, a stopwatch for timing intervals, a pen and data sheet for trial-by-trial entries, and so forth. Relegating such cumbersome and monotonous tasks to the operations of the computer not only relieves boredom, it also saves the time that would be required to teach and practice the procedures.

Another major objective was to improve the chances of obtaining meaningful research outcomes by minimizing noise in the data. With computer-assisted research, errors of measurement can be reduced and errors of procedure virtually eliminated; in addition, various steps can easily be taken to control extraneous variables, even if they would be too complex or onerous for manual procedures.

In designing programs for analyzing data, we confined ourselves to routines for data entry, editing, and statistical calculations; we purposely excluded routines for drawing graphs even though they are common in professional research. In line with principles described earlier, we deemed it important that students actively manipulate the data and experience the outcome firsthand. Given the introductory level of the course, this purpose would not be served well by manual statistical calculations, which often fail to provide much insight into the meaning of the statistics, particularly when computational formulas are used in place of defining formulas. By comparison, graphing can be an aesthetically pleasing exercise that uncovers relations among variables that otherwise might be overlooked. Furthermore, in our experience, it is the rare student who truly understands graphs such as scatter plots or frequency distributions without constructing a few.

We were careful to provide each program with the same "look and feel." The system of menus is straightforward, if not flashy, and field testing over the first two semesters provided the opportunity to eliminate bugs and to increase 
user friendliness. As a consequence, a single training session is usually sufficient to allow even computer-naive students to use the system with relative independence.

\section{ILLUSTRATIONS}

The software's success can be judged best if one considers the projects that it allows the students to conduct and the quality of the resulting data. What follows is a synopsis of the projects currently in use, along with illustrative results obtained by the students.

\section{Psychometric Theory}

The first project teaches basic concepts in observation and measurement. The students work in groups of three; one serves as the subject and the others as observers. The subject's task is to use the joystick to track a randomly moving target on the video screen. At the outset, the target appears at the center of the screen, and a message prompts the subject to move the cursor onto the target. The trial begins when the subject does so. The target normally appears as the outline of a square, but when the subject places the cursor on it, the square changes color. The session is divided into 20 trials lasting $40 \mathrm{sec}$ each. During the intertrial intervals, the observers record their independent estimates of the time spent "on target." At the end, the computer displays the actual times. The students use these data to construct scatter plots showing the relation between the various pairs of the trial-by-trial time estimates. They also calculate Pearson's $r$ and the formulas of the least-squares regression lines, which they then superimpose on the scatterplots.

The several data sets obtained in each section supply interesting lessons on reliability and validity. Consider Figure 1, in which the results from one group are presented. The left panel shows the correlation between the estimates of the two observers; interobserver reliability is surprisingly high, considering that no training was given. Unfortunately, reliability does not imply validity, and this point is driven home by the rest of Figure 1. The center panel shows the correlation between the first observer and the computer; obviously, the observer's estimates have no connection with the actual time on target. The right panel shows that the second observer did slightly better. Besides providing students with first-hand experience in the assessment of reliability and validity, this exercise engenders healthy skepticism about the credibility of data collected by unaided human observers.

\section{Psychophysics}

In the second project, the students measure the strength of the Müller-Lyer illusion by using the psychophysical method of adjustment. The students work in pairs; each one takes a turn as subject while the other looks on. The session consists of 40 trials, divided equally between the Müller-Lyer figure and a control figure. The Müller-Lyer figure consists of a single horizontal line with arrowheads pointing to the left at each end and a right-pointing arrowhead in between. The subject uses the joystick to slide the right arrowhead to what is judged to be the middle of the line; pushing a button on the joystick housing registers the judgment. The control figure is identical, except that the arrowheads are replaced with vertical lines.

Several precautions ensure that the subject does not use extraneous cues to decide the position of the sliding stimulus: the Müller-Lyer and control figures alternate randomly across trials; the screen position of each figure varies across trials; the starting position of the sliding stimulus varies, sometimes appearing on the left and sometimes on the right; and judgments must be registered within $10 \mathrm{sec}$ of the trial's onset (incomplete trials are repeated at the end). The use of the joystick also is important; if the sliding stimulus were positioned by tapping a key, subjects could adopt a counting strategy for fixing its position.

With such controls, subjects reliably misjudge the length of the line in the Müller-Lyer figure. In the top panel of Figure 2 are data from an individual subject, in the form of cumulative frequency distributions of judgment errors. The difference in judgments on the Müller-Lyer and control trials is striking, with no overlap in the distributions. The students construct figures such as this to find the me-
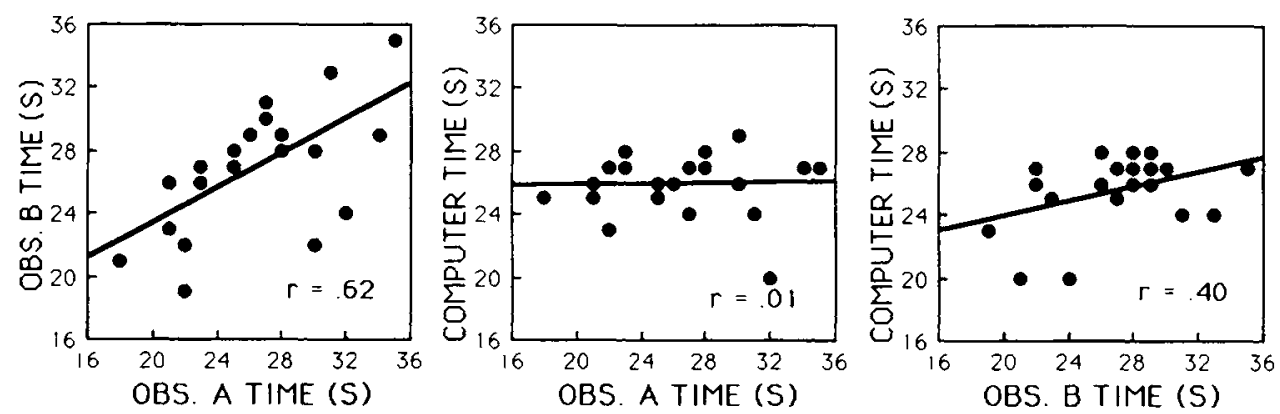

Figure 1. Left: Correlation between two observers' estimates of a subject's time on target in 20 trials of a stimulus-tracking task. Center: Correlation between the first observer's estimates and the actual time on tanget as recorded by computer. Right: Correlation between the second observer's estimates and the computer records. 

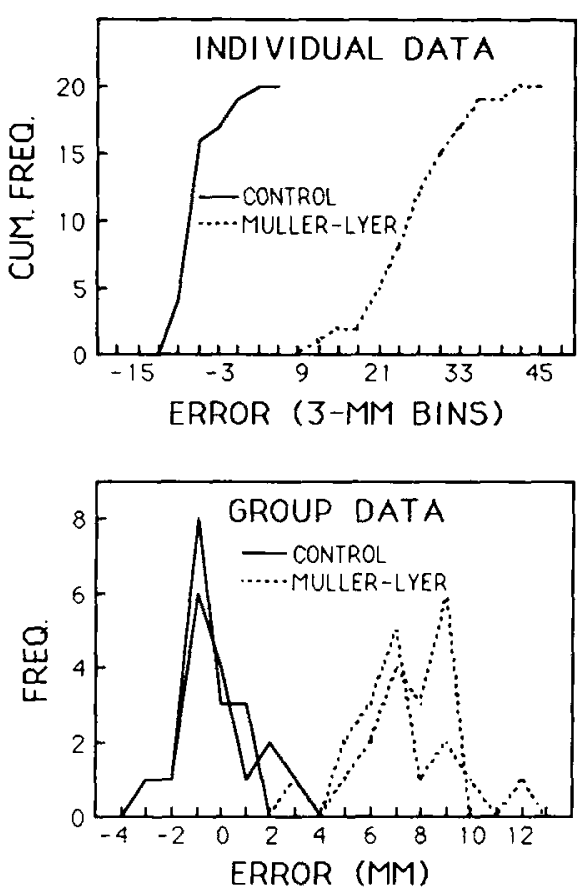

Figure 2. Top: Cumulative frequency distributions of an individuals errors in judging the center of Müller-Lyer and control figures. Data are expressed in deviations from the objective center in millimeters; numbers on the abscissa designate the upper limits of 3-mm class intervals. Bottom: Frequency distributions of median errors with the Müller-Lyer and control figures. Each distribution is based on data from 16 subjects exposed to both conditions. Data for one pair of distributions were collected in 1988; data for the other, in 1989.

dian errors for each subject. The resulting pairs of medians (16 per laboratory section) are grouped for analysis. In the bottom panel of Figure 2, illustrative group data are shown as frequency distributions of the median errors. The two distributions for each condition represent two sections of 16 students who took the course a year apart; together, they illustrate the reliability of the effect.

\section{Operant Conditioning}

The changes in behavior that can be brought about by contingencies of reinforcement are among the most impressive demonstrations in scientific psychology, particularly when students conduct the research with laboratory animals. Unfortunately, economic constraints, political factors, and ethical concerns (for some) combine to make the use of animals in undergraduate courses increasingly difficult. A number of programs allow research with simulated animals (e.g., Bainbridge, 1986), but Goolkasian (1989) reports that student reaction to simulations is negative. Another solution is to use human subjects, but it is difficult to identify manipulations that are effective in the absence of the considerable control typically exerted in the animal case.

In our operant conditioning project, the students themselves are the subjects in an experiment concerned with temporal contingencies of reinforcement (cf. Baron, Menich, \& Perone, 1983). The baseline condition involves a series of disjunctive reaction time trials in which feedback is contingent on correct responses. Each correct response produces the word "Good" and the awarding of a point; incorrect responses produce the word "Wrong," without a point. In the experimental condition, feedback is presented only if the responses are correct and occur within a time limit. Responses outside the limit simply produce the message "Your response was too slow." The time limit depends on the subject's behavior. Initially it is set at the 75th percentile of the reaction times during the last 10 baseline trials; the limit is adjusted in similar fashion every 10 trials thereafterunless the new limit would be less stringent, in which case the old one is retained.

The effect of this temporal contingency is evaluated in a multiple-baseline-across-subjects design. Each pair of students constitutes a separate experiment: for one, the contingency is imposed after a baseline of 80 trials; for the other, the baseline is 160 trials. This design allows practice and other carry-over effects to be distinguished from the effects of the independent variable, in the context of the small- $N$ approach common within operant psychology. Results from one pair are shown in Figure 3, expressed in terms of mean response speeds per block of 10 trials. The contingency increased speeds in both subjects. Although this effect is surprisingly robust, not every pair produces results that are this clear, and there are occasional failures, at least with respect to response speed. But additional analyses of reinforcement rates and error rates almost always show clear differences. The analysis

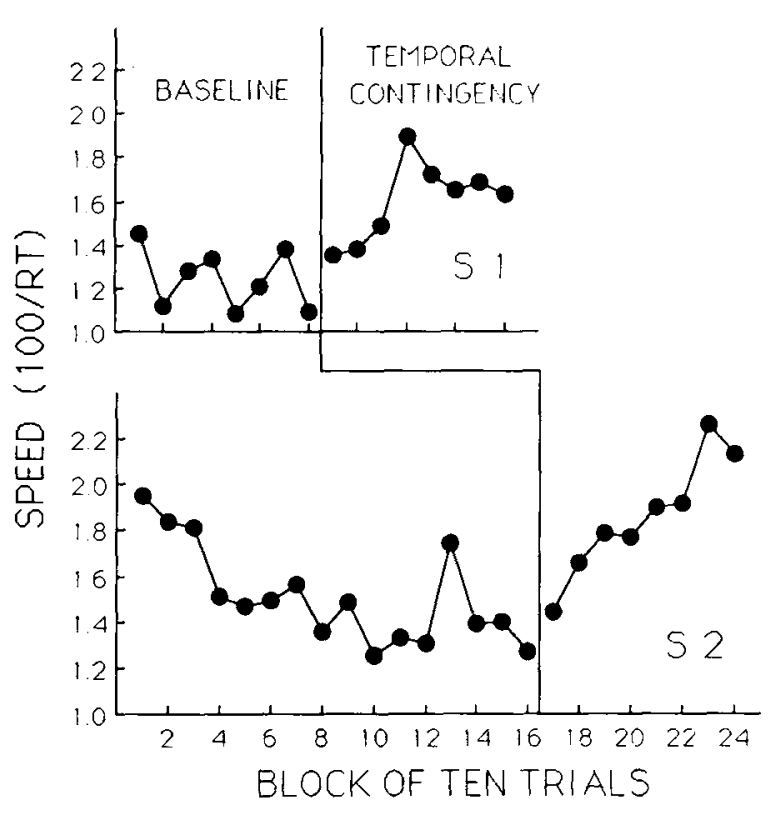

Figure 3. Response speeds of 2 subjects before and after imposition of a temporal contingency of reinforcement. 
of errors is especially interesting, because it provides the basis for discussing speed-accuracy tradeoffs.

\section{Perceptual Adaptation}

The last project is a computerized extension of Stratton's $(1896,1897)$ classic study of adaptation to distorted visual fields. Stratton wore goggles that inverted the field horizontally and vertically, and his actions were often the opposite of what a situation demanded-for example, he reached upward when attempting to grasp an object located below. Over the course of 3 days, however, Stratton began to function more effectively. In the class experiment, the students are exposed to a similar, albeit more limited, type of distortion-one in which they must exercise visuomotor skills that have reversed consequences.

At the beginning of each trial, the video screen displays 42 squares arranged in the pattern of a five-point star. The subject's task is to connect the squares by drawing a line with the joystick. Distortion is introduced by manipulating the relation between movement of the joystick and movement of the line in the horizontal and vertical planes. Thus, a $2 \times 2$ factorial design is created by crossing conditions of normal and reversed movement in the horizontal plane with conditions of normal and reversed movement in the vertical plane.

Elements of the task are similar to those used in mirror tracing procedures, but this version has some advantages. The 10 trials are standardized by requiring the subject to move the cursor into a designated starting square and then pressing a joystick button to begin. Next, an arrow prompts the subject to connect the squares in clockwise order. Each square is darkened as it is connected. If the subject skips a square, a warning sounds, a corrective prompt appears ("Fill boxes in order"), and the square remains open. These steps ensure that the procedure is followed by every subject and that the time required to trace the star is measured precisely. Also worth noting is that separate manipulations in the vertical and horizontal planes are not possible with standard mirror tracing stands, which, unlike computers, are limited by the physics of light.

The students are assigned randomly to the four cells of the design, and the data are combined across several sections for analysis. Data from 52 students are shown in Figure 4. The left panel shows the mean time to complete the first two trials. There is a clear and statistically significant interaction between the distortions in the horizontal and in the vertical planes: The extent to which performance is slowed by distortion in either plane depends on the condition in the other plane-if one type of distortion is present, adding the other type makes no further difference. The right panel shows data from the last two trials. Although there is substantial adaptation, the interaction remains.

This project reliably generates a factorial interaction; the interaction can be detected readily in graphs of the cell means; and the graphic interpretation corresponds to the outcome of analysis of variance. This happy combination of features allows students to learn about factorial experiments in a tidy context that minimizes distractions and maximizes the opportunity to grasp central issues of design, analysis, and interpretation.

\section{STUDENT EVALUATIONS}

Evaluations of our course have been positive. As part of an extensive questionnaire given at the end of the last five semesters, students were asked to rate their agreement with the following statement: "The lab projects helped me to learn about research methods in psychology." The scale was anchored at the positive end by a score of 5 ("almost always") and at the negative end by a 1 ("almost never"). As can be seen in the left panel of Figure 5, the median ratings have been consistently high (black circles). Responses to a parallel statement about the lecture part of the course have also been positive, at least in the most recent semesters (open circles). The students also indicated whether the laboratory projects

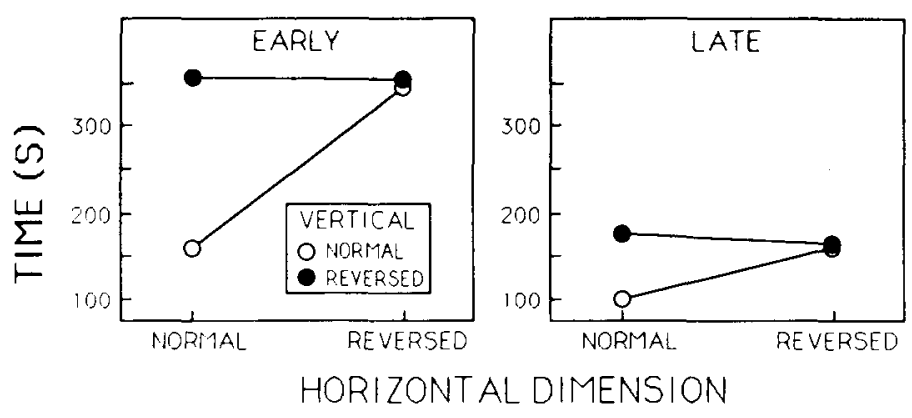

Figure 4. Mean time to complete two trials in the star-tracing procedure, under conditions in which normal or reversed consequences of joystick movements were arranged in the horizontal and vertical planes. Left: Trials 1 and 2. Right: Trials 9 and 10. 

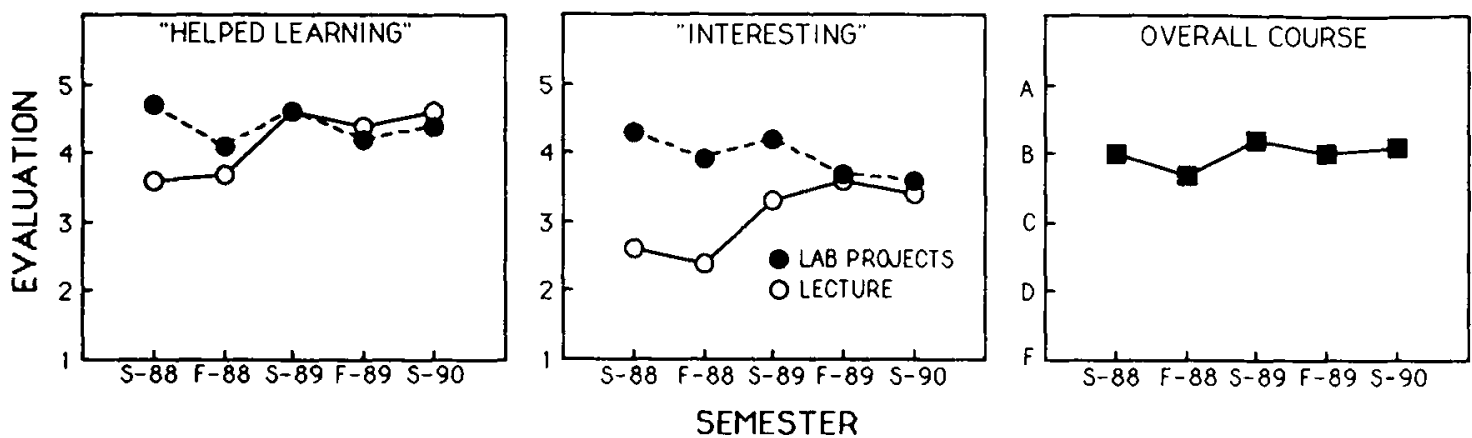

Figure 5. Median student ratings of the methodology course, in response to items about whether the laboratory projects and lectures helped learning (left) and whether they were interesting (center), and about the overall quality of the course (right). Higher scores represent more favorable ratings.

and lectures were interesting. As can be seen in the center panel, responses to the laboratory were favorable in this respect as well, but the lectures did not capture much interest until recently. Finally, the students were asked to rate the overall course by assigning a conventional letter grade. As can be seen in the right panel of Figure 5, the ratings have been remarkably stable at the grade of " $B$," which is defined as "very good" on the questionnaire. It is not possible, of course, to attribute these reactions to the use of the computers. Nevertheless, it is reassuring that the reactions have been so favorable.

\section{CONCLUSIONS}

Although the course that we offer is conventional in both objectives and format, the use of computer-assisted research projects in the laboratory sections provides significant benefits. Computers and locally developed software duplicate the functions of a well-equipped traditional laboratory, at a fraction of the cost. Data are collected quickly and efficiently, leaving more time for analysis and discussion while increasing the number and complexity of the projects that can be conducted. Procedural errors are virtually eliminated, measurement errors are reduced, and various control procedures are implemented without burdensome requirements on students. As a consequence, uncontrolled variability is minimized, and the opportunity for meaningful research outcomes is maximized. The laboratory's menu of projects can be expanded by writing additional software in high-level languages, without the expense of additional hardware. Of course, should suitable commercial software become available, it could be adopted as well.

\section{REFERENCES}

BAINBRIDGE, W. S. (1986). Experiments in psychology: A microcomputer laboratory [Computer program]. Belmont, CA: Wadsworth.

Baron, A., Menich, S. R., Perone, M. (1983), Reaction times of younger and older men and temporal contingencies of reinforcement. Journal of the Experimental Analysis of Behavior, 40, 275-287.

EAMON, D. B. (1986). Integrating a computer component into the student psychology laboratory: Problems and prospects. Behavior Research Methods, Instruments, \& Computers, 18, 245-250.

Goolkasian, P. (1989). Computerized laboratories for psychology instruction: How successful are they? Behavior Research Methods, instruments, \& Computers, 21, 148-150.

STratTon, G. (1896). Some preliminary experiments in vision without inversion of the retinal image. Psychological Review, 3, 611-617.

Stratton, G. (1897). Vision without inversion of the retinal image. Psychological Review, 4, 341-360, 463-481. 